\title{
Zeros of Jacobi-Sobolev orthogonal polynomials following non-coherent pair of measures
}

\author{
ELIANA X.L. DE ANDRADE ${ }^{1}$, CLEONICE F. BRACCIALI ${ }^{1}$, \\ MIRELA V. DE MELLO ${ }^{1}$ and TERESA E. PÉREZ ${ }^{2}$ \\ ${ }^{1}$ DCCE, IBILCE, UNESP - Universidade Estadual Paulista \\ 15054-000 São José do Rio Preto, SP, Brazil \\ ${ }^{2}$ Departamento de Matemática Aplicada and \\ Instituto Carlos I de Física Teórica y Computacional \\ Universidad de Granada, 18071 Granada, Spain \\ E-mails: eliana@ibilce.unesp.br / cleonice@ibilce.unesp.br / \\ mirela_vanina@yahoo.com.br/tperez@ugr.es
}

\begin{abstract}
Zeros of orthogonal polynomials associated with two different Sobolev inner products involving the Jacobi measure are studied. In particular, each of these Sobolev inner products involves a pair of closely related Jacobi measures. The measures of the inner products considered are beyond the concept of coherent pairs of measures. Existence, real character, location and interlacing properties for the zeros of these Jacobi-Sobolev orthogonal polynomials are deduced.
\end{abstract}

Mathematical subject classification: 33C45, 33C47, 26C10.

Key words: Sobolev orthogonal polynomials, Jacobi orthogonal polynomials, Zeros of orthogonal polynomials.

\section{Introduction}

Consider the inner product

$$
\langle f, g\rangle_{S}=\sum_{j=0}^{k} \int_{\mathbb{R}} f^{(j)}(x) g^{(j)}(x) d \psi_{j}(x), \quad k \geq 1,
$$


where $d \psi_{j}$, for $j=0,1, \ldots, k$, are positive measures supported on $\mathbb{R}$. This inner product is known as Sobolev inner product and the associated sequence of monic orthogonal polynomials, $\left\{S_{n}\right\}_{n=0}^{\infty}$, is known as a sequence of monic Sobolev orthogonal polynomials.

This kind of inner product is non-standard in the sense that the shift operator, i.e., the multiplication operator by $x$, is not self-adjoint

$$
\langle x f, g\rangle_{S} \neq\langle f, x g\rangle_{S},
$$

where $f$ and $g$ are polynomials with real coefficients. Therefore, some of the usual properties of standard orthogonal polynomials are not true. In fact, the usual three term recurrence relation and the properties about the zeros (real and simple characters, interlacing, etc.) are no longer valid.

In 1991, A. Iserles et al. [9] studied Sobolev inner products as (1) for $k=1$ when the two measures $d \psi_{0}$ and $d \psi_{1}$ are related. If we denote by $\left\{P_{n}^{\psi_{i}}\right\}_{n=0}^{\infty}$ $(i=0,1)$ the sequence of monic orthogonal polynomials with respect to the standard inner product

$$
\langle f, g\rangle_{\psi_{i}}=\int_{\mathbb{R}} f(x) g(x) d \psi_{i}(x), \quad i=0,1,
$$

then $\left\{d \psi_{0}, d \psi_{1}\right\}$ is a coherent pair of measures if

$$
P_{n}^{\psi_{1}}(x)=\frac{1}{n+1}\left[P_{n+1}^{\prime} \psi_{0}(x)+\sigma_{n} P_{n}^{\prime} \psi_{0}(x)\right], \quad \text { for } n \geq 1,
$$

where $\sigma_{n}$ are non-zero constants. As a consequence (see [9]), the sequence of monic Sobolev orthogonal polynomials $\left\{S_{n}\right\}_{n=0}^{\infty}$ associated with the Sobolev inner product

$$
\langle f, g\rangle_{S}=\langle f, g\rangle_{\psi_{0}}+\left\langle f^{\prime}, g^{\prime}\right\rangle_{\psi_{1}}
$$

satisfies

$$
S_{n+1}(x)+a_{n} S_{n}(x)=P_{n+1}^{\psi_{0}}(x)+\sigma_{n} P_{n}^{\psi_{0}}(x), \quad n \geq 1 .
$$

H.G. Meijer [13] has shown that if $\left\{d \psi_{0}, d \psi_{1}\right\}$ is a coherent pair of measures, then both measures are closely related and at least one of them must be classical.

Sobolev orthogonal polynomials associated with coherent pairs have been exhaustively studied. Algebraic and differential properties, as well as properties 
about zeros, have been investigated. In particular, in [6], [8], and [11], several results about existence, location and interlacing properties of the zeros of orthogonal polynomials with respect to Gegenbauer-Sobolev and Hermite-Sobolev inner products are shown. Moreover, in [14], the authors considered special Jacobi-Sobolev and Laguerre-Sobolev inner products, where the pair of measures forms a coherent pair and they proved interlacing properties of the zeros of Sobolev orthogonal polynomials.

In [2] and [3] it has been introduced an alternative approach to study Sobolev inner products such that the corresponding orthogonal polynomials still satisfy a relation of the form (3). This kind of Sobolev inner products generalizes Sobolev inner products defined from a coherent pair of measures and it allows to extend the results about Sobolev orthogonal polynomials beyond the concept of coherent pairs. In [7] the authors have considered the inverse problem: starting from the relation (3) to obtain a pair of quasi-definite moment functionals such that (3) holds. Their results show that the pair of measures involved being coherent is not necessary for (3) to hold.

In the present paper, properties for the zeros of Sobolev orthogonal polynomials associated with inner products of the form (2) have been investigated. The measures of the inner products involve Jacobi measures and they are beyond the concept of coherent pairs of measures.

For $\alpha, \beta>-1$, let $d \psi^{(\alpha, \beta)}$ denotes the classical Jacobi measure on $[-1,1]$ given by

$$
d \psi^{(\alpha, \beta)}(x)=(1-x)^{\alpha}(1+x)^{\beta} d x,
$$

$\left\{P_{n}^{(\alpha, \beta)}\right\}_{n=0}^{\infty}$ be the sequence of classical monic Jacobi orthogonal polynomials and $\rho_{n}^{(\alpha, \beta)}=\left\langle P_{n}^{(\alpha, \beta)}, P_{n}^{(\alpha, \beta)}\right\rangle_{\psi^{(\alpha, \beta)} \text {. }}$.

It is well known that the zeros of $P_{n}^{(\alpha, \beta)}$ are all real, distinct and lie inside $(-1,1)$. We denote the zeros of $P_{n}^{(\alpha, \beta)}$ by $p_{n, i}^{(\alpha, \beta)}, i=1,2, \ldots, n$, in increasing order. For more details about these polynomials see, for instance, [5] and [15].

In this paper we consider two Sobolev inner products given in [3], namely

Type I. For $\left|\kappa_{1}\right| \leq 1, \quad \kappa_{2} \geq\left|\kappa_{3}\right|$,

$$
\begin{aligned}
& d \psi_{0}=\left(1+\kappa_{1} x\right) d \psi^{(\alpha, \beta)}(x), \\
& d \psi_{1}=\left(\kappa_{2}+\kappa_{3} x\right) d \psi^{(\alpha+1, \beta+1)}(x) .
\end{aligned}
$$


Type II. For $\left|\kappa_{3}\right| \geq 1, \quad \kappa_{2}, \kappa_{4} \geq 0, \quad \kappa_{1} \geq-\left|\kappa_{3}\right| \kappa_{2} /\left(1+\left|\kappa_{3}\right|\right)$,

$$
\begin{aligned}
& d \psi_{0}=d \psi^{(\alpha, \beta)}(x), \\
& d \psi_{1}=\left(\kappa_{1}+\frac{\kappa_{2} \kappa_{3}}{\kappa_{3}-x}\right) d \psi^{(\alpha+1, \beta+1)}(x)+\kappa_{2} \kappa_{4} \delta\left(\kappa_{3}\right) .
\end{aligned}
$$

When $\kappa_{1} \neq 0, \kappa_{2}>0$ and $\kappa_{3}=0$, the measures involved in type I form a coherent pair (see [13]). In [14], the authors have proved that the zeros of the corresponding Sobolev orthogonal polynomials are real and simple, and they have interlacing properties. When $\kappa_{3} \neq 0$, the pair of measures no longer forms a coherent pair and, in this case, we will show in Section 2 that, under some conditions, the interlacing properties established in [14] for $\kappa_{3}=0$ still hold even when $\kappa_{3} \neq 0$.

When $\kappa_{1}=0$ the pair of measures of type II is also a coherent pair of measures (see [13]). In this case, properties about zeros for the corresponding Sobolev orthogonal appear in [14]. In [10] the authors have studied the special case when $\kappa_{1}=\kappa_{4}=0, \kappa_{2}>0$ and $\kappa_{3}=-1$. When $\kappa_{1} \neq 0$, the pair of measures of type II no longer forms a coherent pair. Section 3 is devoted to show interlacing properties for the zeros of Jacobi-Sobolev orthogonal polynomials of type II when $\kappa_{1} \neq 0$. Indeed, our results generalize the interlacing properties established in [14] for the particular case $\kappa_{1}=0$.

In [1] it has been studied properties of the zeros of orthogonal polynomials with respect to Gegenbauer-Sobolev inner product where the associated pair of measures does not form a symmetrically coherent pair.

\section{Jacobi-Sobolev inner product of type I}

Let us consider the following modification of the Jacobi weight

$$
d \psi^{\left(\alpha, \beta, \kappa_{1}\right)}(x)=\left(1+\kappa_{1} x\right) d \psi^{(\alpha, \beta)}(x)=\left(1+\kappa_{1} x\right)(1-x)^{\alpha}(1+x)^{\beta} d x,
$$

defined on $[-1,1]$, where $\left|\kappa_{1}\right| \leq 1$. We denote by $\left\{P_{n}^{\left(\alpha, \beta, \kappa_{1}\right)}\right\}_{n=0}^{\infty}$ the sequence of monic orthogonal polynomials associated with $d \psi^{\left(\alpha, \beta, \kappa_{1}\right)}$, and

$$
\rho_{n}^{\left(\alpha, \beta, \kappa_{1}\right)}=\int_{-1}^{1}\left(P_{n}^{\left(\alpha, \beta, \kappa_{1}\right)}(x)\right)^{2}\left(1+\kappa_{1} x\right)(1-x)^{\alpha}(1+x)^{\beta} d x .
$$


The following result is known (see, for instance, [3])

$$
P_{n}^{(\alpha, \beta)}(x)=P_{n}^{\left(\alpha, \beta, \kappa_{1}\right)}(x)+d_{n-1} P_{n-1}^{\left(\alpha, \beta, \kappa_{1}\right)}(x), \quad n \geq 1,
$$

where

$$
d_{n-1}=\frac{\kappa_{1} \rho_{n}^{(\alpha, \beta)}}{\rho_{n-1}^{\left(\alpha, \beta, \kappa_{1}\right)}}, \quad n \geq 1 .
$$

Observe that $\operatorname{sgn}\left(d_{n}\right)=\operatorname{sgn}\left(\kappa_{1}\right)$ where, as usual,

$$
\operatorname{sgn}(a)=\left\{\begin{array}{cl}
|a| / a, & \text { if } a \neq 0, \\
0, & \text { if } a=0 .
\end{array}\right.
$$

For $n \geq 1$, the zeros of $P_{n}^{\left(\alpha, \beta, \kappa_{1}\right)}$ are real, simple and all lie in the interval $(-1,1)$. If we denote their zeros by $p_{n, i}^{\left(\alpha, \beta, \kappa_{1}\right)}, i=1,2, \ldots, n$, in increasing order, then they interlace with the zeros of the classical Jacobi polynomials and their position depends on the sign of $\kappa_{1}$ (see [4]):

for $-1 \leq \kappa_{1}<0$ and $1 \leq i \leq n-1$,

$$
-1<p_{n, i}^{\left(\alpha, \beta, \kappa_{1}\right)}<p_{n, i}^{(\alpha, \beta)}<p_{n-1, i}^{\left(\alpha, \beta, \kappa_{1}\right)}<p_{n, i+1}^{\left(\alpha, \beta, \kappa_{1}\right)}<p_{n, i+1}^{(\alpha, \beta)}<1,
$$

and for $0<\kappa_{1} \leq 1$ and $1 \leq i \leq n-1$,

$$
-1<p_{n, i}^{(\alpha, \beta)}<p_{n, i}^{\left(\alpha, \beta, \kappa_{1}\right)}<p_{n-1, i}^{\left(\alpha, \beta, \kappa_{1}\right)}<p_{n, i+1}^{(\alpha, \beta)}<p_{n, i+1}^{\left(\alpha, \beta, \kappa_{1}\right)}<1 .
$$

Consider the following Sobolev inner product, introduced in [3],

$$
\begin{aligned}
\langle f, g\rangle_{J S_{1}}= & \int_{-1}^{1} f(x) g(x)\left(1+\kappa_{1} x\right) d \psi^{(\alpha, \beta)}(x) \\
& +\int_{-1}^{1} f^{\prime}(x) g^{\prime}(x)\left(\kappa_{2}+\kappa_{3} x\right) d \psi^{(\alpha+1, \beta+1)}(x),
\end{aligned}
$$

with $\left|\kappa_{1}\right| \leq 1$ and $\kappa_{2} \geq\left|\kappa_{3}\right|$. Then $\langle\cdot, \cdot\rangle_{J S_{1}}$ is positive definite and we will refer to it as Jacobi-Sobolev inner product of type $I$.

Let $\left\{S_{n}^{J_{1}}\right\}_{n=0}^{\infty}$ denote the sequence of monic orthogonal polynomials with respect to (7), we will refer to it as sequence of Jacobi-Sobolev orthogonal polynomials of type $I$. In addition, we denote $\rho_{n}^{J_{1}}=\left\langle S_{n}^{J_{1}}, S_{n}^{J_{1}}\right\rangle_{J S_{1}}$. 
Furthermore, $S_{0}^{J_{1}}(x)=1$ and for $n \geq 0$

$$
S_{n+1}^{J_{1}}(x)+a_{n}^{J_{1}} S_{n}^{J_{1}}(x)=P_{n+1}^{(\alpha, \beta)}(x)=P_{n+1}^{\left(\alpha, \beta, \kappa_{1}\right)}(x)+d_{n} P_{n}^{\left(\alpha, \beta, \kappa_{1}\right)}(x),
$$

where $a_{n}^{J_{1}}$ is given by the expression ([3])

$$
a_{n}^{J_{1}}=\frac{\left(\kappa_{1}+n(n+\alpha+\beta+2) \kappa_{3}\right) \rho_{n+1}^{(\alpha, \beta)}}{\rho_{n}^{J_{1}}} .
$$

Observe that, if $\kappa_{1}=0$, then $\operatorname{sgn}\left(a_{n}^{J_{1}}\right)=\operatorname{sgn}\left(\kappa_{3}\right)$. Otherwise, if $\operatorname{sgn}\left(\kappa_{1}\right)=$ $\operatorname{sgn}\left(\kappa_{3}\right)$, then $\operatorname{sgn}\left(a_{n}^{J_{1}}\right)=\operatorname{sgn}\left(\kappa_{1}\right)=\operatorname{sgn}\left(\kappa_{3}\right)$.

\subsection{Zeros of $S_{n}^{J_{1}}$ and $P_{n}^{(\alpha, \beta)}$}

For $n, i \geq 0$ we define

$$
m_{i, n}^{J_{1}}=\int_{-1}^{1} S_{n}^{J_{1}}(x)\left[x+\operatorname{sgn}\left(\kappa_{3}\right)\right]^{i} d \psi^{(\alpha, \beta)}(x),
$$

and

$$
\hat{m}_{i, n}^{J_{1}}=\int_{-1}^{1} S_{n}^{\prime J_{1}}(x)\left[x+\operatorname{sgn}\left(\kappa_{3}\right)\right]^{i} d \psi^{(\alpha+1, \beta+1)}(x) .
$$

Since $\left\langle S_{n}^{J_{1}},\left[x+\operatorname{sgn}\left(\kappa_{3}\right)\right]^{i}\right\rangle_{J S_{1}}=0$ for $n \geq 1$ and $0 \leq i \leq n-1$, we obtain

$$
\kappa_{1} m_{i+1, n}^{J_{1}}+\left(1-\operatorname{sgn}\left(\kappa_{3}\right) \kappa_{1}\right) m_{i, n}^{J_{1}}=-i\left(\kappa_{3} \hat{m}_{i, n}^{J_{1}}+\left(\kappa_{2}-\left|\kappa_{3}\right|\right) \hat{m}_{i-1, n}^{J_{1}}\right) \text {. }
$$

Using integration by parts in (10) we have for $n \geq 1$ and $i \geq 0$,

$$
\hat{m}_{i, n}^{J_{1}}=(i+\alpha+\beta+2) m_{i+1, n}^{J_{1}}-\left(2 \operatorname{sgn}\left(\kappa_{3}\right) i+\eta\right) m_{i, n}^{J_{1}},
$$

where $\eta=(\beta+1)\left[1+\operatorname{sgn}\left(\kappa_{3}\right)\right]-(\alpha+1)\left[1-\operatorname{sgn}\left(\kappa_{3}\right)\right]$. Since

$$
\eta=\left\{\begin{aligned}
-2(\alpha+1), & \text { if } \kappa_{3}<0, \\
2(\beta+1), & \text { if } \kappa_{3}>0,
\end{aligned}\right.
$$

then $\operatorname{sgn}(\eta)=\operatorname{sgn}\left(\kappa_{3}\right)$.

Substituting (12) in (11), the following three term recurrence relation holds

$$
m_{i+1, n}^{J_{1}}=-\frac{1}{\kappa_{1}+i \kappa_{3}(i+\alpha+\beta+2)}\left(A_{i} m_{i, n}^{J_{1}}-B_{i} m_{i-1, n}^{J_{1}}\right),
$$


for $n \geq 2$ and $1 \leq i \leq n-1$, where

$$
\begin{aligned}
& \left.A_{i}=\left(\kappa_{2}-3\left|\kappa_{3}\right|\right) i^{2}+\left[\left(\kappa_{2}-\left|\kappa_{3}\right|\right)(\alpha+\beta+1)-\kappa_{3} \eta\right)\right] i+\left(1-\operatorname{sgn}\left(\kappa_{3}\right) \kappa_{1}\right), \\
& B_{i}=\left(\kappa_{2}-\left|\kappa_{3}\right|\right)\left[2 \operatorname{sgn}\left(\kappa_{3}\right)(i-1)+\eta\right] i
\end{aligned}
$$

Since $\kappa_{2} \geq\left|\kappa_{3}\right|$ and $\operatorname{sgn}(\eta)=\operatorname{sgn}\left(\kappa_{3}\right)$, we observe that $\operatorname{sgn}\left(B_{i}\right)=\operatorname{sgn}\left(\kappa_{3}\right)$ for $i \geq 1$.

Moreover, in order to assure the positivity of $A_{i}$ for $i \geq 1$, we need some additional conditions.

Lemma 2.1. Suppose $\alpha, \beta \geq 0, \kappa_{2} \geq 3\left|\kappa_{3}\right|$ and $\operatorname{sgn}\left(\kappa_{1}\right)=\operatorname{sgn}\left(\kappa_{3}\right)$ if $\kappa_{1} \neq 0$. Then $A_{i}>0$ for $i \geq 1$.

Proof. Observe that we can write

$$
A_{i}=\left[\kappa_{2}-3\left|\kappa_{3}\right|\right] i^{2}+\left[\left(\kappa_{2}-3\left|\kappa_{3}\right|\right)(\alpha+\beta+1)-2 \kappa_{3} \beta\right] i+\left(1-\left|\kappa_{1}\right|\right)
$$

for $\kappa_{3}<0$, and

$$
A_{i}=\left[\kappa_{2}-3\left|\kappa_{3}\right|\right] i^{2}+\left[\left(\kappa_{2}-3\left|\kappa_{3}\right|\right)(\alpha+\beta+1)+2 \kappa_{3} \alpha\right] i+\left(1-\left|\kappa_{1}\right|\right)
$$

for $\kappa_{3}>0$. Then, the result holds.

Lemma 2.2. Assume that the conditions of Lemma 2.1 hold. For $n \geq 1$, if $\kappa_{1}=0$, we get

$$
m_{0, n}^{J_{1}}=0, \quad \operatorname{sgn}\left(m_{i, n}^{J_{1}}\right)=(-1)^{n+i}\left[\operatorname{sgn}\left(\kappa_{3}\right)\right]^{n+i}, \quad 1 \leq i \leq n,
$$

and if $\kappa_{1} \neq 0$

$$
\operatorname{sgn}\left(m_{i, n}^{J_{1}}\right)=(-1)^{n+i}\left[\operatorname{sgn}\left(\kappa_{3}\right)\right]^{n+i}, \quad 0 \leq i \leq n .
$$

Proof. Suppose $\kappa_{1}=0$. From (11), we get $m_{0, n}^{J_{1}}=0, n \geq 1$. On the other hand, using the well known property for the monic classical Jacobi polynomials (see [15])

$$
P_{n}^{\prime(\alpha, \beta)}(x)=n P_{n-1}^{(\alpha+1, \beta+1)}(x), \quad n \geq 0,
$$


in relation (8), we have

$$
\hat{m}_{0, n}^{J_{1}}=-a_{n-1}^{J_{1}} \int_{-1}^{1} S_{n-1}^{J_{1}}(x) d \psi^{(\alpha+1, \beta+1)}(x) .
$$

Therefore

$$
\hat{m}_{0, n}^{J_{1}}=(-1)^{n-1} a_{n-1}^{J_{1}} a_{n-2}^{J_{1}} \ldots a_{2}^{J_{1}} a_{1}^{J_{1}} \int_{-1}^{1} S_{1}^{\prime J_{1}}(x) d \psi^{(\alpha+1, \beta+1)}(x) .
$$

Since $\operatorname{sgn}\left(a_{n}^{J_{1}}\right)=\operatorname{sgn}\left(\kappa_{3}\right)$ and $\int_{-1}^{1} S_{1}^{\prime J_{1}}(x) d \psi^{(\alpha+1, \beta+1)}(x)>0$, we deduce

$$
\operatorname{sgn}\left(\hat{m}_{0, n}^{J_{1}}\right)=(-1)^{n+1}\left[\operatorname{sgn}\left(\kappa_{3}\right)\right]^{n+1}, \quad n \geq 1 .
$$

By making $i=0$ in (12), we get $\hat{m}_{0, n}^{J_{1}}=(\alpha+\beta+2) m_{1, n}^{J_{1}}$, and then

$$
\operatorname{sgn}\left(m_{1, n}^{J_{1}}\right)=\operatorname{sgn}\left(\hat{m}_{0, n}^{J_{1}}\right)=(-1)^{n+1}\left[\operatorname{sgn}\left(\kappa_{3}\right)\right]^{n+1}, \quad n \geq 1 .
$$

Now, suppose that $\kappa_{1} \neq 0$. Using (8), we obtain

$$
\int_{-1}^{1} S_{n+1}^{J_{1}}(x) d \psi^{(\alpha, \beta)}(x)+a_{n}^{J_{1}} \int_{-1}^{1} S_{n}^{J_{1}}(x) d \psi^{(\alpha, \beta)}(x)=0, \quad n \geq 0,
$$

and then $m_{0, n+1}^{J_{1}}=-a_{n}^{J_{1}} m_{0, n}^{J_{1}}$. Since $m_{0,0}^{J_{1}}=\int_{-1}^{1} S_{0}^{J_{1}}(x) d \psi^{(\alpha, \beta)}(x)>0$ for $n \geq 0$, we get

$$
\operatorname{sgn}\left(m_{0, n}^{J_{1}}\right)=(-1)^{n} \operatorname{sgn}\left(a_{n-1}^{J_{1}} a_{n-2}^{J_{1}} \cdots a_{1}^{J_{1}}\right) \operatorname{sgn}\left(m_{0,0}^{J_{1}}\right)=(-1)^{n}\left[\operatorname{sgn}\left(\kappa_{3}\right)\right]^{n} .
$$

The substitution of $i=0$ in (13) yields $m_{1, n}^{J_{1}}=-\left(1-\operatorname{sgn}\left(\kappa_{3}\right) \kappa_{1}\right) m_{0, n}^{J_{1}} / \kappa_{1}$, then

$$
\operatorname{sgn}\left(m_{1, n}^{J_{1}}\right)=(-1)^{n+1}\left[\operatorname{sgn}\left(\kappa_{3}\right)\right]^{n} \operatorname{sgn}\left(\kappa_{1}\right)=(-1)^{n+1}\left[\operatorname{sgn}\left(\kappa_{3}\right)\right]^{n+1} .
$$

Therefore, using mathematical induction on $i$ in (13), we get the result.

Lemma 2.3. Under the hypotheses of Lemma 2.1, let $\pi_{r}(x)$ be a monic polynomial of degree $r$, with $1 \leq r \leq n$, such that all of its zeros are real and lie in $[-1,1]$. Let us define

$$
I_{r, n}=\int_{-1}^{1} S_{n}^{J_{1}}(x) \pi_{r}(x) d \psi^{(\alpha, \beta)}(x) .
$$

Then $\operatorname{sgn}\left(I_{r, n}\right)=(-1)^{n+r}\left[\operatorname{sgn}\left(\kappa_{3}\right)\right]^{n+r}$. 
Proof. Let $-1 \leq t_{r, 1} \leq t_{r, 2} \leq \cdots \leq t_{r, r} \leq 1$ be the zeros of $\pi_{r}(x)$. Then

$$
\pi_{r}(x)=\prod_{j=1}^{r}\left\{x+\operatorname{sgn}\left(\kappa_{3}\right)-\left[t_{r, j}+\operatorname{sgn}\left(\kappa_{3}\right)\right]\right\}=\sum_{i=0}^{r} c_{i}\left[x+\operatorname{sgn}\left(\kappa_{3}\right)\right]^{i},
$$

where $c_{r}=1$ and, if $c_{i} \neq 0$, then $\operatorname{sgn}\left(c_{i}\right)=(-1)^{r-i}\left[\operatorname{sgn}\left(\kappa_{3}\right)\right]^{r-i}, i=$ $0,1, \ldots, r$. Using (9),

$$
I_{r, n}=\sum_{i=0}^{r} c_{i} m_{i, n}^{J_{1}},
$$

and the result holds from Lemma 2.2.

Now we will show that, under the hypotheses of Lemma 2.1, the $n$-th JacobiSobolev orthogonal polynomial of type I, $S_{n}^{J_{1}}$, has $n$ real and simple zeros and they interlace with the zeros of the classical Jacobi polynomial $P_{n}^{(\alpha, \beta)}$.

Theorem 2.4. Suppose that the conditions of Lemma 2.1 hold. Then, for $n \geq 2, S_{n}^{J_{1}}$ has $n$ real and simple zeros. If we denote $s_{n, i}^{J_{1}}$ for $1 \leq i \leq n$, the zeros of $S_{n}^{J_{1}}$ in increasing order, then they satisfy

for $\kappa_{3}<0$,

$$
s_{n, i}^{J_{1}}<p_{n, i}^{(\alpha, \beta)}<s_{n, i+1}^{J_{1}}<p_{n, i+1}^{(\alpha, \beta)}, \quad 1 \leq i \leq n-1,
$$

for $\kappa_{3}>0$,

$$
p_{n, i}^{(\alpha, \beta)}<s_{n, i}^{J_{1}}<p_{n, i+1}^{(\alpha, \beta)}<s_{n, i+1}^{J_{1}}, \quad 1 \leq i \leq n-1 .
$$

Proof. Define

$$
\pi_{n-1}^{j}(x)=\frac{P_{n}^{(\alpha, \beta)}(x)}{x-p_{n, j}^{(\alpha, \beta)}}=\prod_{i=1, i \neq j}^{n}\left(x-p_{n, i}^{(\alpha, \beta)}\right), \quad 1 \leq j \leq n .
$$

Then, $\operatorname{deg} \pi_{n-1}^{j}=n-1$ and, using Lemma 2.3, we get

$$
\operatorname{sgn}\left(I_{n-1, n}^{j}\right)=(-1)^{2 n-1}\left[\operatorname{sgn}\left(\kappa_{3}\right)\right]^{2 n-1}=-\operatorname{sgn}\left(\kappa_{3}\right),
$$

where

$$
I_{n-1, n}^{j}=\int_{-1}^{1} S_{n}^{J_{1}}(x) \pi_{n-1}^{j}(x) d \psi^{(\alpha, \beta)}(x) .
$$


Applying the Gaussian quadrature rule based on the $n$ zeros of $P_{n}^{(\alpha, \beta)}$, we obtain

$$
\begin{aligned}
I_{n-1, n}^{j} & =\sum_{i=1}^{n} w_{n, i} S_{n}^{J_{1}}\left(p_{n, i}^{(\alpha, \beta)}\right) \pi_{n-1}^{j}\left(p_{n, i}^{(\alpha, \beta)}\right) \\
& =w_{n, j} S_{n}^{J_{1}}\left(p_{n, j}^{(\alpha, \beta)}\right) P_{n}^{\prime(\alpha, \beta)}\left(p_{n, j}^{(\alpha, \beta)}\right)
\end{aligned}
$$

for $j=1,2, \ldots, n$.

Suppose $\kappa_{3}<0$. In this case, for $j=1,2, \ldots, n$, we get

$$
S_{n}^{J_{1}}\left(p_{n, j}^{(\alpha, \beta)}\right) P_{n}^{\prime(\alpha, \beta)}\left(p_{n, j}^{(\alpha, \beta)}\right)>0 .
$$

Therefore, there is just one zero of $S_{n}^{J_{1}}$ in each interval $\left(p_{n, i-1}^{(\alpha, \beta)}, p_{n, i}^{(\alpha, \beta)}\right)$ for $i=2,3, \ldots, n$. Since $P_{n}^{(\alpha, \beta)}$ and $S_{n}^{J_{1}}$ are monic, there is one zero of $S_{n}^{J_{1}}$ in $\left(-\infty, p_{n, 1}^{(\alpha, \beta)}\right)$. Then, (14) holds.

For $\kappa_{3}>0$, we observe that

$$
S_{n}^{J_{1}}\left(p_{n, j}^{(\alpha, \beta)}\right) P_{n}^{\prime(\alpha, \beta)}\left(p_{n, j}^{(\alpha, \beta)}\right)<0, \quad \text { for } \quad j=1,2, \ldots, n,
$$

and a similar argument as above shows (15).

Moreover, it is possible to show interlacing properties between the zeros of Jacobi-Sobolev orthogonal polynomials, $S_{n}^{J_{1}}$, and the zeros of the classical Jacobi polynomials $P_{n}^{(\alpha, \beta+1)}, P_{n}^{(\alpha+1, \beta)}$ and $P_{n}^{(\alpha+1, \beta+1)}$.

Theorem 2.5. Under the hypotheses of Lemma 2.1, for $n \geq 2$ and $1 \leq i \leq$ $n-1$, we get

i) the zeros of $S_{n}^{J_{1}}$ interlace with the zeros of $P_{n}^{(\alpha, \beta+1)}$ and $P_{n}^{(\alpha+1, \beta)}$ as follows

$$
\begin{aligned}
& s_{n, i}^{J_{1}}<p_{n, i}^{(\alpha, \beta+1)}<s_{n, i+1}^{J_{1}}<p_{n, i+1}^{(\alpha, \beta+1)}<1, \\
& -1<p_{n, i}^{(\alpha+1, \beta)}<s_{n, i}^{J_{1}}<p_{n, i+1}^{(\alpha+1, \beta)}<s_{n, i+1}^{J_{1}},
\end{aligned}
$$

ii) the zeros of $P_{n-1}^{(\alpha+1, \beta+1)}$ separate the zeros of $S_{n}^{J_{1}}$ in the following way

$$
s_{n, i}^{J_{1}}<p_{n-1, i}^{(\alpha+1, \beta+1)}<s_{n, i+1}^{J_{1}} \text {. }
$$


Proof. Defining

$$
\pi_{n}^{j}(x)=(x+1) \frac{P_{n}^{(\alpha, \beta+1)}(x)}{x-p_{n, j}^{(\alpha, \beta+1)}}=(x+1) \prod_{i=1, i \neq j}^{n}\left(x-p_{n, i}^{(\alpha, \beta+1)}\right),
$$

and

$$
\hat{\pi}_{n}^{j}(x)=(x-1) \frac{P_{n}^{(\alpha+1, \beta)}(x)}{x-p_{n, j}^{(\alpha+1, \beta)}}=(x-1) \prod_{i=1, i \neq j}^{n}\left(x-p_{n, i}^{(\alpha+1, \beta)}\right),
$$

a similar argument used for Theorem 2.4 shows the result i).

To get the result ii), take

$$
\tilde{\pi}_{n}^{j}(x)=\frac{\left(x^{2}-1\right) P_{n-1}^{(\alpha+1, \beta+1)}(x)}{x-p_{n-1, j}^{(\alpha+1, \beta+1)}}=\left(x^{2}-1\right) \prod_{i=1, i \neq j}^{n-1}\left(x-p_{n-1, i}^{(\alpha+1, \beta+1)}\right)
$$

in Lemma 2.3. Then one obtains

$$
w_{n-1, j} S_{n}^{J_{1}}\left(p_{n-1, j}^{(\alpha+1, \beta+1)}\right) P_{n-1}^{\prime(\alpha+1, \beta+1)}\left(p_{n-1, j}^{(\alpha+1, \beta+1)}\right)<0,
$$

for $j=1,2, \ldots, n-1$.

As a consequence of this theorem, the following result is established.

Corollary 2.6. For $n \geq 1$, Jacobi-Sobolev orthogonal polynomial $S_{n}^{J_{1}}$ has $n$ real and simple zeros inside $(-1,1)$.

Finally, interlacing properties of the zeros of Jacobi-Sobolev orthogonal polynomials of two consecutive degrees can be shown.

Theorem 2.7. Under conditions of Lemma 2.1, for $n \geq 2$, the $n-1$ zeros of $S_{n-1}^{J_{1}}$ interlace with the zeros of $S_{n}^{J_{1}}$ as follows

$$
s_{n, i}^{J_{1}}<s_{n-1, i}^{J_{1}}<s_{n, i+1}^{J_{1}}, \quad 1 \leq i \leq n-1 .
$$

Proof. From (8) we get

$$
a_{n-1}^{J_{1}} S_{n-1}^{J_{1}}\left(s_{n, i}^{J_{1}}\right)=P_{n}^{(\alpha, \beta)}\left(s_{n, i}^{J_{1}}\right), \quad \text { for } \quad i=1,2, \cdots, n .
$$


On the other hand, Theorem 2.4 provides

$$
\operatorname{sgn}\left(S_{n}^{J_{1}}\left(p_{n, i}^{(\alpha, \beta)}\right) P_{n}^{\prime(\alpha, \beta)}\left(p_{n, i}^{(\alpha, \beta)}\right)\right)=-\operatorname{sgn}\left(\kappa_{3}\right),
$$

and then

$$
\operatorname{sgn}\left(S_{n}^{J_{1}^{\prime}}\left(s_{n, i}^{J_{1}}\right) P_{n}^{(\alpha, \beta)}\left(s_{n, i}^{J_{1}}\right)\right)=\operatorname{sgn}\left(\kappa_{3}\right) .
$$

Since $\operatorname{sgn}\left(a_{n-1}^{J_{1}}\right)=\operatorname{sgn}\left(\kappa_{3}\right)$, we deduce $S_{n}^{J_{1^{\prime}}}\left(s_{n, i}^{J_{1}}\right) S_{n-1}^{J_{1}}\left(s_{n, i}^{J_{1}}\right)>0$.

Therefore, $S_{n-1}^{J_{1}}$ has a zero in each interval $\left(s_{n, i-1}^{J_{1}}, s_{n, i}^{J_{1}}\right)$, for $i=2,3, \ldots$, $n$.

Remark 2.8. If we have $\operatorname{sgn}\left(\kappa_{1}\right) \neq \operatorname{sgn}\left(\kappa_{3}\right)$ instead of $\operatorname{sgn}\left(\kappa_{1}\right)=\operatorname{sgn}\left(\kappa_{3}\right)$ in the hypotheses of Lemma 2.1, then $\operatorname{sgn}\left(a_{0}^{J_{1}}\right)=\operatorname{sgn}\left(\kappa_{1}\right)$ and there exists $N \in \mathbb{N}$ such that

$$
\operatorname{sgn}\left(a_{n}^{J_{1}}\right)=\operatorname{sgn}\left(\kappa_{1}+n(n+\alpha+\beta+2) \kappa_{3}\right)=\left\{\begin{array}{l}
\operatorname{sgn}\left(\kappa_{1}\right), \text { if } n<N, \\
\operatorname{sgn}\left(\kappa_{3}\right), \text { if } n \geq N, \text { or } a_{N}^{J_{1}}=0 .
\end{array}\right.
$$

Numerical experiments allow us to conjecture that, also in this case, the zeros of $S_{n}^{J_{1}}$ interlace with the zeros of $P_{n}^{(\alpha, \beta)}$. Moreover, for $1 \leq i \leq n$,

- if $\kappa_{1}<0$ and $\kappa_{3}>0$, then $s_{n, i}^{J_{1}}<p_{n, i}^{(\alpha, \beta)}$ for $n \leq N$ and $p_{n, i}^{(\alpha, \beta)}<s_{n, i}^{J_{1}}$ for $n>N$,

- if $\kappa_{1}>0$ and $\kappa_{3}<0$, then $p_{n, i}^{(\alpha, \beta)}<s_{n, i}^{J_{1}}$ for $n \leq N$ and $s_{n, i}^{J_{1}}<p_{n, i}^{(\alpha, \beta)}$ for $n>N$.

Table 1 describes an example of this fact. Notice, from (8), that if $a_{N}^{J_{1}}=0$ then $S_{N+1}^{J_{1}}(x)=P_{N+1}^{(\alpha, \beta)}(x)$.

\subsection{Zeros of $S_{n}^{J_{1}}$ and $P_{n}^{\left(\alpha, \beta, \kappa_{1}\right)}$}

In this section we relate the zeros of Jacobi-Sobolev orthogonal polynomial of type I with the zeros of the polynomial $P_{n}^{\left(\alpha, \beta, \kappa_{1}\right)}, \kappa_{1} \neq 0$, orthogonal with respect to the first measure in (7).

For $n, i \geq 0$, we define

$$
\mu_{i, n}^{J_{1}}=\int_{-1}^{1} S_{n}^{J_{1}}(x)\left(1+\kappa_{1} x\right)^{i} d \psi^{(\alpha, \beta)}(x),
$$




\begin{tabular}{|c|c|c|}
\hline$i$ & $s_{4, i}^{J_{1}}$ & $p_{4, i}^{(\alpha, \beta)}$ \\
\hline 1 & -0.4308679926 & -0.4307826553 \\
\hline 2 & 0.0148759928 & 0.0149809768 \\
\hline 3 & 0.4375627876 & 0.4376478562 \\
\hline 4 & 0.7781122439 & 0.7781538222 \\
\hline \hline & $s_{4, i}^{J_{1}}<p_{4, i}^{(\alpha, \beta)}, i=1,2,3,4$. \\
\hline
\end{tabular}

\begin{tabular}{|c|c|c|}
\hline$i$ & $s_{5, i}^{J_{1}}$ & $p_{5, i}^{(\alpha, \beta)}$ \\
\hline 1 & -0.5449824458 & -0.5449890822 \\
\hline 2 & -0.1698341048 & -0.1698432787 \\
\hline 3 & 0.2118047152 & 0.2117956865 \\
\hline 4 & 0.5577706724 & 0.5577641564 \\
\hline 5 & 0.8276284403 & 0.8276254591 \\
\hline \hline & $p_{5, i}^{(\alpha, \beta)}<s_{5, i}^{J_{1}}, i=1,2,3,4,5$. \\
\hline
\end{tabular}

Table 1 - Zeros of polynomials $S_{n}^{J_{1}}$ and $P_{n}^{(\alpha, \beta)}$ for $n=4,5, \alpha=2, \beta=5, \kappa_{1}=-0.999$, $\kappa_{2}=5$, and $\kappa_{3}=0.02$. Here $N=4$.

and

$$
\hat{\mu}_{i, n}^{J_{1}}=\int_{-1}^{1} S_{n}^{\prime J_{1}}(x)\left(1+\kappa_{1} x\right)^{i} d \psi^{(\alpha+1, \beta+1)}(x) .
$$

Observe that, since $\left\langle S_{n}^{J_{1}},\left(1+\kappa_{1} x\right)^{i}\right\rangle_{J S_{1}}=0$, for $n \geq 1$,

$$
\mu_{i+1, n}^{J_{1}}=-i \kappa_{3} \hat{\mu}_{i, n}^{J_{1}}+i\left(\kappa_{3}-\kappa_{1} \kappa_{2}\right) \hat{\mu}_{i-1, n}^{J_{1}}, \quad i=0,1, \ldots, n-1 .
$$

On the other hand, using integration by parts we get

$$
\begin{aligned}
\kappa_{1} \hat{\mu}_{i, n}^{J_{1}}= & (i+2+\alpha+\beta) \mu_{i+1, n}^{J_{1}} \\
& -\left[2 i+(\alpha+1)\left(1-\kappa_{1}\right)+(\beta+1)\left(1+\kappa_{1}\right)\right] \mu_{i, n}^{J_{1}} \\
& +i\left(1-\kappa_{1}^{2}\right) \mu_{i-1, n}^{J_{1}}
\end{aligned}
$$

for $n \geq 1$ and $i \geq 0$. Then, the following recurrence relation holds

$$
\mu_{i+1, n}^{J_{1}}=-\frac{\kappa_{1}}{\kappa_{1}+i \kappa_{3}(i+2+\alpha+\beta)}\left(A_{i} \mu_{i, n}^{J_{1}}-B_{i} \mu_{i-1, n}^{J_{1}}+C_{i} \mu_{i-2, n}^{J_{1}}\right),
$$

for $n \geq 1$ and $i=1,2, \ldots, n-1$, where

$$
\begin{aligned}
& A_{i}=\left(\kappa_{2}-3 \frac{\kappa_{3}}{\kappa_{1}}\right) i^{2}+\left(\kappa_{2}(\alpha+\beta+1)-\frac{\kappa_{3}}{\kappa_{1}}\left[\alpha\left(2-\kappa_{1}\right)+\beta\left(2+\kappa_{1}\right)+3\right]\right) i \\
& B_{i}=\left(2 \kappa_{2}-\frac{\kappa_{3}}{\kappa_{1}}\left(3-\kappa^{2}\right)\right) i^{2}+\left(\kappa_{2}-\frac{\kappa_{3}}{\kappa_{1}}\right)\left(\alpha\left(1-\kappa_{1}\right)+\beta\left(1+\kappa_{1}\right)\right) i \\
& C_{i}=\left(\kappa_{2}-\frac{\kappa_{3}}{\kappa_{1}}\right)\left(1-\kappa_{1}^{2}\right) i(i-1) .
\end{aligned}
$$


In order to obtain $A_{i}, B_{i}$ and $C_{i}$ as non-negative coefficients, we need some additional conditions. Observe that the conditions given in the next lemma are sufficient.

Lemma 2.9. Suppose $\alpha, \beta \geq 0, \kappa_{2} \geq 3 \kappa_{3} / \kappa_{1} \geq 0$ and $\operatorname{sgn}\left(\kappa_{1}\right)=\operatorname{sgn}\left(\kappa_{3}\right)$. Then $A_{i}, B_{i}$ and $C_{i}$ are non-negative for $i \geq 1$.

A similar argument used in Lemma 2.2 allows us to obtain the sign of $\mu_{i, n}^{J_{1}}$ and $\hat{\mu}_{i, n}^{J_{1}}$, using the hypotheses of Lemma 2.9.

Lemma 2.10. Assume that the conditions of Lemma 2.9 hold. For $n \geq 1$, we have
i) $\mu_{1, n}^{J_{1}}=0, \quad \operatorname{sgn}\left(\mu_{i, n}^{J_{1}}\right)=(-1)^{n+i}\left[\operatorname{sgn}\left(\kappa_{1}\right)\right]^{n}, \quad i=2,3, \ldots, n$,
ii) $\operatorname{sgn}\left(\hat{\mu}_{i, n}^{J_{1}}\right)=(-1)^{n+i+1}\left[\operatorname{sgn}\left(\kappa_{1}\right)\right]^{n+1}, \quad i=0,1, \ldots, n-1$.

The next lemma is analogous to Lemma 2.3 and it can be proved using the same technique. Again, we assume that the hypotheses of Lemma 2.9 are valid.

Lemma 2.11. Under conditions of Lemma 2.9, for $n \geq 2$ it follows

i) Let $\pi_{r}$ be a monic polynomial of degree $r, 1 \leq r \leq n-1$, such that all of its zeros are real and lie in $(-1,1)$. Define

$$
I_{r, n}=\frac{1}{\kappa_{1}} \int_{-1}^{1} S_{n}^{J_{1}}(x) \pi_{r}(x) d \psi^{\left(\alpha, \beta, \kappa_{1}\right)}(x),
$$

then $\operatorname{sgn}\left(I_{r, n}\right)=(-1)^{n+r+1}\left[\operatorname{sgn}\left(\kappa_{1}\right)\right]^{n+r+1}$.

ii) Let $\pi_{r}$ be a monic polynomial of degree $r, 1 \leq r \leq n$, with all real zeros in $(-1,1)$. If we define

$$
J_{r, n}=\frac{1}{\kappa_{1}} \int_{-1}^{1} S_{n}^{\prime J_{1}}(x) \pi_{r}(x) d \psi^{\left(\alpha+1, \beta+1, \kappa_{1}\right)}(x),
$$

then $\operatorname{sgn}\left(J_{r, n}\right)=(-1)^{n+r+1}\left[\operatorname{sgn}\left(\kappa_{1}\right)\right]^{n+r}$. 
Proof. To prove i), let $-1<t_{r, 1} \leq t_{r, 2} \leq \cdots \leq t_{r, r}<1$ be the zeros of $\pi_{r}$. Then

$$
\pi_{r}(x)=\prod_{j=1}^{r}\left(x-t_{r, j}\right)=\prod_{j=1}^{r}\left(x+\frac{1}{\kappa_{1}}-\left(t_{r, j}+\frac{1}{\kappa_{1}}\right)\right)=\sum_{i=0}^{r} c_{i}\left(x+\frac{1}{\kappa_{1}}\right)^{i},
$$

with $c_{r}=1$. When $-1 \leq \kappa_{1}<0$ we have $t_{r, j}+\frac{1}{\kappa_{1}}<0$ and when $0<\kappa_{1} \leq 1$ we have $t_{r, j}+\frac{1}{\kappa_{1}}>0$. Therefore

$$
\operatorname{sgn}\left(c_{i}\right)=(-1)^{r-i}\left[\operatorname{sgn}\left(\kappa_{1}\right)\right]^{r-i}, \quad i=0,1, \ldots, r .
$$

Hence,

$$
I_{r, n}=\sum_{i=0}^{r} \frac{c_{i}}{\kappa_{1}^{i+1}} \int_{-1}^{1} S_{n}^{J_{1}}(x)\left(1+\kappa_{1} x\right)^{i+1} d \psi^{(\alpha, \beta)}(x)=\sum_{i=0}^{r} \frac{c_{i}}{\kappa_{1}^{i+1}} \mu_{i+1, n}^{J_{1}},
$$

and using Lemma 2.10 the result holds. A similar argument shows ii).

Now, we have the necessary tools to get the announced interlacing property between the zeros of $S_{n}^{J_{1}}$ and the zeros of $P_{n}^{\left(\alpha, \beta, \kappa_{1}\right)}$.

Theorem 2.12. Under the conditions of Lemma 2.9, for $n \geq 2$ and $1 \leq i \leq$ $n-1$, the zeros of $S_{n}^{J_{1}}$ satisfy

i) If $-1 \leq \kappa_{1}<0$,

$$
p_{n, i}^{\left(\alpha, \beta, \kappa_{1}\right)}<s_{n, i}^{J_{1}}<p_{n, i+1}^{\left(\alpha, \beta, \kappa_{1}\right)}<s_{n, i+1}^{J_{1}},
$$

and, if $0<\kappa_{1} \leq 1$,

$$
s_{n, i}^{J_{1}}<p_{n, i}^{\left(\alpha, \beta, \kappa_{1}\right)}<s_{n, i+1}^{J_{1}}<p_{n, i+1}^{\left(\alpha, \beta, \kappa_{1}\right)} .
$$

ii) The zeros of $P_{n-1}^{\left(\alpha, \beta, \kappa_{1}\right)}$ separate the zeros of $S_{n}^{J_{1}}$. That is,

$$
s_{n, i}^{J_{1}}<p_{n-1, i}^{\left(\alpha, \beta, \kappa_{1}\right)}<s_{n, i+1}^{J_{1}} .
$$

Collecting all the interlacing properties given in (5), (6) and Theorems 2.4 and 2.12, we have for $-1 \leq \kappa_{1}<0$,

$$
p_{n, i}^{\left(\alpha, \beta, \kappa_{1}\right)}<s_{n, i}^{J_{1}}<p_{n, i}^{(\alpha, \beta)}<p_{n-1, i}^{\left(\alpha, \beta, \kappa_{1}\right)}<p_{n, i+1}^{\left(\alpha, \beta, \kappa_{1}\right)}, \quad 1 \leq i \leq n-1,
$$


and, for $0<\kappa_{1} \leq 1$,

$$
p_{n, i}^{(\alpha, \beta)}<s_{n, i}^{J_{1}}<p_{n, i}^{\left(\alpha, \beta, \kappa_{1}\right)}<p_{n-1, i}^{\left(\alpha, \beta, \kappa_{1}\right)}<p_{n, i+1}^{(\alpha, \beta)}, \quad 1 \leq i \leq n-1 .
$$

To finish this section, the extremal points of $S_{n}^{J_{1}}$ can be analyzed. Denote the extremal points of $S_{n}^{J_{1}}$ by $\hat{s}_{n, i}^{J_{1}}, i=1,2, \ldots, n-1$, in increasing order.

Theorem 2.13. Under the hypotheses of Lemma 2.9, for $n \geq 3$ the polynomial $S_{n}^{J_{1}}$ has $n-1$ extremal points in the interval $(-1,1)$ and they satisfy

$$
p_{n, i}^{\left(\alpha+1, \beta+1, \kappa_{1}\right)}<\hat{s}_{n, i}^{J_{1}}<p_{n, i+1}^{\left(\alpha+1, \beta+1, \kappa_{1}\right)}, \quad 1 \leq i \leq n-1 .
$$

\section{Jacobi-Sobolev inner product of type II}

Let $d \psi(x)$ be the measure defined on $[-1,1]$ by means of

$$
\langle f, g\rangle_{\psi}=\int_{-1}^{1} f(x) g(x) \frac{\kappa_{3}}{\kappa_{3}-x}(1-x)^{\alpha+1}(1+x)^{\beta+1} d x+\kappa_{4} f\left(\kappa_{3}\right) g\left(\kappa_{3}\right),
$$

where $\left|\kappa_{3}\right| \geq 1$ and $\kappa_{4} \geq 0$, and let $\left\{P_{n}^{\left(\alpha, \beta, \kappa_{3}, \kappa_{4}\right)}\right\}_{n=0}^{\infty}$ be the corresponding sequence of monic orthogonal polynomials. In Maroni [12] (see also [3]), the author has obtained the relation

$$
P_{n}^{\left(\alpha, \beta, \kappa_{3}, \kappa_{4}\right)}(x)=P_{n}^{(\alpha+1, \beta+1)}(x)+d_{n-1} P_{n-1}^{(\alpha+1, \beta+1)}(x), \quad n \geq 1,
$$

where

$$
d_{n-1}=-\frac{\rho_{n}^{\left(\alpha, \beta, \kappa_{3}, \kappa_{4}\right)}}{\kappa_{3} \rho_{n-1}^{(\alpha+1, \beta+1)}}, \quad \rho_{n}^{\left(\alpha, \beta, \kappa_{3}, \kappa_{4}\right)}=\left\langle P_{n}^{\left(\alpha, \beta, \kappa_{3}, \kappa_{4}\right)}, P_{n}^{\left(\alpha, \beta, \kappa_{3}, \kappa_{4}\right)}\right\rangle_{\psi} .
$$

Note that $\operatorname{sgn}\left(d_{n-1}\right)=-\operatorname{sgn}\left(\kappa_{3}\right)$.

In this section we consider Jacobi-Sobolev inner product of type II, introduced in [3], given by the expression

$$
\begin{aligned}
& \langle f, g\rangle_{J S_{2}}=\int_{-1}^{1} f(x) g(x) d \psi^{(\alpha, \beta)}(x) \\
+ & \int_{-1}^{1} f^{\prime}(x) g^{\prime}(x)\left(\kappa_{1}+\frac{\kappa_{2} \kappa_{3}}{\kappa_{3}-x}\right) d \psi^{(\alpha+1, \beta+1)}(x)+\kappa_{2} \kappa_{4} f^{\prime}\left(\kappa_{3}\right) g^{\prime}\left(\kappa_{3}\right),
\end{aligned}
$$


where

$$
\left|\kappa_{3}\right| \geq 1, \quad \kappa_{2}, \kappa_{4} \geq 0 \quad \text { and } \quad \kappa_{1} \geq-\frac{\left|\kappa_{3}\right| \kappa_{2}}{1+\left|\kappa_{3}\right|} .
$$

We denote by $\left\{S_{n}^{J_{2}}\right\}_{n=0}^{\infty}$ the sequence of monic orthogonal polynomials associated with $\langle\cdot, \cdot\rangle_{J S_{2}}$, and we call it sequence of monic Jacobi-Sobolev orthogonal polynomials of type II. These polynomials satisfy $S_{0}^{J_{2}}(x)=1, S_{1}^{J_{2}}(x)=$ $P_{1}^{(\alpha, \beta)}(x)$ and

$$
S_{n+1}^{J_{2}}(x)+a_{n}^{J_{2}} S_{n}^{J_{2}}(x)=P_{n+1}^{(\alpha, \beta)}(x)+b_{n} P_{n}^{(\alpha, \beta)}(x), \quad n \geq 1,
$$

where

$$
a_{n}^{J_{2}}=\frac{\left(\rho_{n}^{(\alpha, \beta)}+\kappa_{1} n^{2} \rho_{n-1}^{(\alpha+1, \beta+1)}\right) b_{n}}{\rho_{n}^{J_{2}}}, \quad n \geq 1,
$$

$\rho_{n}^{J_{2}}=\left\langle S_{n}^{J_{2}}, S_{n}^{J_{2}}\right\rangle_{J S_{2}}$ and

$$
b_{n}=\frac{n+1}{n} d_{n-1}=-\frac{n+1}{n} \frac{\rho_{n}^{\left(\alpha, \beta, \kappa_{3}, \kappa_{4}\right)}}{\kappa_{3} \rho_{n-1}^{(\alpha+1, \beta+1)}}, \quad n \geq 1 .
$$

Since $\rho_{n-1}^{(\alpha+1, \beta+1)}=(n+\alpha+\beta+1) \rho_{n}^{(\alpha, \beta)} / n$, we can also write

$$
a_{n}^{J_{2}}=\frac{\left(1+\kappa_{1} n(n+\alpha+\beta+1)\right) b_{n} \rho_{n}^{(\alpha, \beta)}}{\rho_{n}^{J_{2}}} .
$$

Observe that $\operatorname{sgn}\left(b_{n}\right)=\operatorname{sgn}\left(d_{n-1}\right)=-\operatorname{sgn}\left(\kappa_{3}\right)$ and, if $\kappa_{1}>0$, then $\operatorname{sgn}\left(a_{n}^{J_{2}}\right)=$ $\operatorname{sgn}\left(b_{n}\right)=-\operatorname{sgn}\left(\kappa_{3}\right)$.

\subsection{Zeros of $S_{n}^{J_{2}}$ and $P_{n}^{(\alpha, \beta)}$}

For $n, i \geq 0$, we define

$$
v_{i, n}^{J_{2}}=\int_{-1}^{1} S_{n}^{J_{2}}(x)\left(x-\kappa_{3}\right)^{i} d \psi^{(\alpha, \beta)}(x)
$$

and

$$
\begin{aligned}
\hat{v}_{i, n}^{J_{2}} & =\int_{-1}^{1} S_{n}^{\prime J_{2}}(x)\left(x-\kappa_{3}\right)^{i} \frac{\kappa_{3}-x}{\kappa_{3}} d \psi(x) \\
& =\int_{-1}^{1} S_{n}^{\prime J_{2}}(x)\left(x-\kappa_{3}\right)^{i}(1-x)^{\alpha+1}(1+x)^{\beta+1} d x
\end{aligned}
$$


Because of the orthogonality property, we have $\left\langle S_{n}^{J_{2}},\left(x-\kappa_{3}\right)^{i}\right\rangle_{J S_{2}}=0$, for $n \geq 1$, and then

$$
v_{i, n}^{J_{2}}=i\left(-\kappa_{1} \hat{v}_{i-1, n}^{J_{2}}+\kappa_{3} \kappa_{2} \hat{v}_{i-2, n}^{J_{2}}\right), \quad i \geq 2 .
$$

Integration by parts in (22) for $n \geq 1$ and $i \geq 1$ provides

$$
\begin{aligned}
\hat{v}_{i, n}^{J_{2}}= & (i+2+\alpha+\beta) v_{i+1, n}^{J_{2}} \\
& +\left[2 \kappa_{3} i+(\alpha+1)\left(1+\kappa_{3}\right)-(\beta+1)\left(1-\kappa_{3}\right)\right] v_{i, n}^{J_{2}} \\
& +i\left(\kappa_{3}^{2}-1\right) v_{i-1, n}^{J_{2}},
\end{aligned}
$$

and then, if we define $v_{-1, n}^{J_{2}}=0$, for $n \geq 1$ and $i=2,3, \ldots, n-1$, the following three term recurrence relation can be deduced

$$
v_{i, n}^{J_{2}}=-\frac{i}{1+\kappa_{1}(i+1+\alpha+\beta) i}\left(A_{i} v_{i-1, n}^{J_{2}}-B_{i} v_{i-2, n}^{J_{2}}+C_{i} v_{i-3, n}^{J_{2}}\right),
$$

where

$$
\begin{aligned}
A_{i} & =-\kappa_{3}\left(\kappa_{2}-2 \kappa_{1}\right) i+\kappa_{1}\left[\kappa_{3}(\alpha+\beta)+\alpha-\beta\right]-\kappa_{3} \kappa_{2}(\alpha+\beta), \\
B_{i} & =\left[2 \kappa_{3}^{2} \kappa_{2}-\kappa_{1}\left(\kappa_{3}^{2}-1\right)\right] i+\kappa_{2}\left[\kappa_{3}^{2}(\alpha+\beta-2)+\kappa_{3}(\alpha-\beta)\right]+\kappa_{1}\left(\kappa_{3}^{2}-1\right), \\
C_{i} & =\kappa_{3} \kappa_{2}\left(1-\kappa_{3}^{2}\right)(i-2) .
\end{aligned}
$$

The next lemma establishes sufficient conditions to determine the sign of the above coefficients.

Lemma 3.1. For $\kappa_{2} \geq 2 \kappa_{1} \geq 0, \alpha+\beta>2$ and

$$
\begin{cases}\alpha \leq \beta, & \text { if } \kappa_{3} \leq-1, \\ \alpha \geq \beta, & \text { if } \kappa_{3} \geq 1,\end{cases}
$$

then $\operatorname{sgn}\left(A_{i}\right)=-\operatorname{sgn}\left(\kappa_{3}\right)$ and $B_{i}>0$, for $i \geq 1$. Moreover, if $\left|\kappa_{3}\right| \neq 1$ then $\operatorname{sgn}\left(C_{i}\right)=-\operatorname{sgn}\left(\kappa_{3}\right)$ and if $\left|\kappa_{3}\right|=1$ then $C_{i}=0$.

We remark that Lemma 3.1 establishes sufficient conditions in order to obtain the sign of $A_{i}, B_{i}$ and $C_{i}$. Under conditions of Lemma 3.1, analogous techniques to those used in Lemmas 2.2 and 2.3 allow us to prove the next two lemmas. 
Lemma 3.2. For $n \geq 3$, we have $v_{0, n}^{J_{2}}=0$ and

$$
\operatorname{sgn}\left(v_{i, n}^{J_{2}}\right)=-\left[\operatorname{sgn}\left(\kappa_{3}\right)\right]^{n+i}, \quad 1 \leq i \leq n-1 .
$$

Lemma 3.3. For $n \geq 3$, let us consider $\pi_{r}$ a monic polynomial of degree $r$, $1 \leq r \leq n-1$, such that all its zeros are real, simple and lie in the interval $(-1,1)$. Define

$$
I_{r, n}=\int_{-1}^{1} S_{n}^{J_{2}}(x) \pi_{r}(x) d \psi^{(\alpha, \beta)}(x) .
$$

Then $\operatorname{sgn}\left(I_{r, n}\right)=-\left[\operatorname{sgn}\left(\kappa_{3}\right)\right]^{n+r}$.

Under the same restrictions given in Lemma 3.1 for the parameters and using the above two lemmas, we can show that the $n$-th Jacobi-Sobolev orthogonal polynomial of type II, $S_{n}^{J_{2}}$, has $n$ different real zeros and at least $n-1$ zeros lie inside $(-1,1)$. We denote the real zeros of $S_{n}^{J_{2}}$, in increasing order, by $s_{n, i}^{J_{2}}$, $i=1,2, \ldots, n$.

Theorem 3.4. Under the conditions of Lemma 3.1, for $n \geq 3, S_{n}^{J_{2}}$ has $n$ real zeros and at least $n-1$ of them lie inside the interval $(-1,1)$. Moreover, denoting the zeros of $S_{n}^{J_{2}}$, by $s_{n, i}^{J_{2}}, i=1,2, \ldots, n$, in increasing order, then

i) if $\kappa_{3} \leq-1$,

$$
s_{n, i}^{J_{2}}<p_{n, i}^{(\alpha, \beta)}<s_{n, i+1}^{J_{2}}<p_{n, i+1}^{(\alpha, \beta)}, \quad 1 \leq i \leq n-1,
$$

if $\kappa_{3} \geq 1$,

$$
p_{n, i}^{(\alpha, \beta)}<s_{n, i}^{J_{2}}<p_{n, i+1}^{(\alpha, \beta)}<s_{n, i+1}^{J_{2}}, \quad 1 \leq i \leq n-1 .
$$

ii) For $1 \leq i \leq n-1$, the following interlacing property holds

$$
s_{n, i}^{J_{2}}<p_{n-1, i}^{(\alpha, \beta)}<s_{n, i+1}^{J_{2}} .
$$

We must point out that one zero of $S_{n}^{J_{2}}$ can be outside the interval $(-1,1)$. Figure 1 shows the graphs of Jacobi-Sobolev orthogonal polynomial of type II $S_{6}^{J_{2}}$ and the classical Jacobi orthogonal polynomial $P_{6}^{(\alpha, \beta)}$. According to Theorem 3.4, in Figure 1(a), with $\kappa_{3}<-1$, we can see that the smallest zero is outside the interval $(-1,1)$. In Figure $1(\mathrm{~b})$, with $\kappa_{3}>1$, we can see that the largest zero is outside $(-1,1)$. 


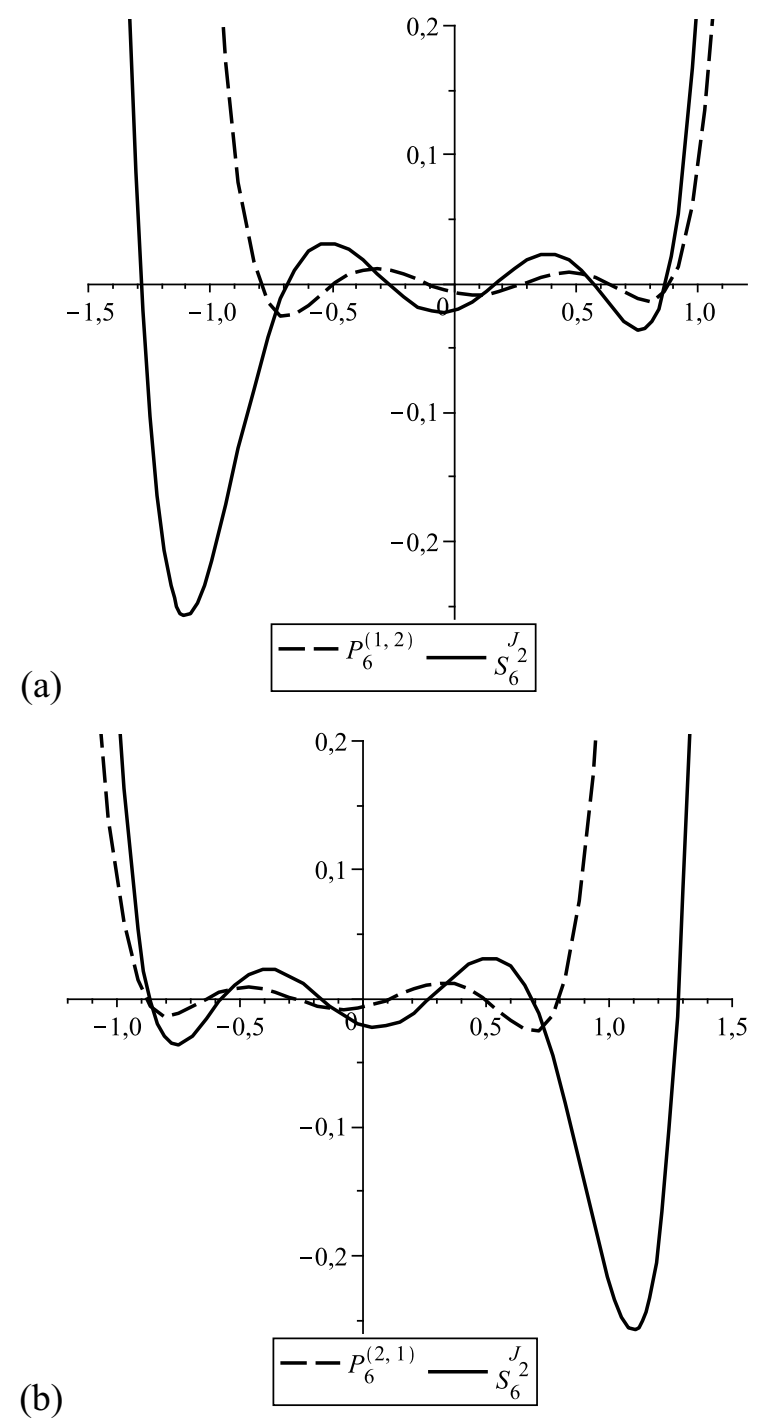

Figure 1 - Graphs of $S_{6}^{J_{2}}$ and $P_{6}^{(\alpha, \beta)}$ with $\kappa_{1}=1, \kappa_{2}=4, \kappa_{4}=1$. (a) $\kappa_{3}=-1.1$, $\alpha=1, \beta=2$. (b) $\kappa_{3}=1.1, \alpha=2, \beta=1$.

\subsection{Some conditions for all zeros of $S_{n}^{J_{2}}$ to lie inside $(-1,1)$}

In this section we obtain some conditions for the parameters in the inner product (18) to assure that all zeros of $S_{n}^{J_{2}}$ lie inside the interval $(-1,1)$. 
We now denote the polynomials $S_{n}^{J_{2}}$ by $S_{n}^{\left(\kappa_{1}, \kappa_{2}, \kappa_{3}, \kappa_{4}\right)}(x)$ and the coefficients $a_{n}^{J_{2}}$ by $a_{n}^{\left(\kappa_{1}, \kappa_{2}, \kappa_{3}, \kappa_{4}\right)}$.

Let $\kappa_{2}$ tend to $\infty$ in $\frac{1}{\kappa_{2}}\langle f, g\rangle_{J S_{2}}$. Then we find that the monic polynomials, $S_{n}^{\left(\kappa_{1}, \infty, \kappa_{3}, \kappa_{4}\right)}(x)$, must satisfy

$$
\int_{-1}^{1} S_{m}^{\prime\left(\kappa_{1}, \infty, \kappa_{3}, \kappa_{4}\right)}(x) S_{n}^{\prime\left(\kappa_{1}, \infty, \kappa_{3}, \kappa_{4}\right)}(x) d \psi(x)=0, \quad \text { for } m \neq n .
$$

Since $\rho_{n}^{J_{2}}>n^{2} \kappa_{2} \rho_{n-1}^{\left(\alpha, \beta, \kappa_{3}, \kappa_{4}\right)}$ and, for a fixed $\kappa_{1}, \operatorname{sgn}\left(a_{n}^{\left(\kappa_{1}, \kappa_{2}, \kappa_{3}, \kappa_{4}\right)}\right)=-\operatorname{sgn}\left(\kappa_{3}\right)$, from (20) we verify that $a_{n}^{\left(\kappa_{1}, \infty, \kappa_{3}, \kappa_{4}\right)}=0$. Then we conclude from (19) that

$$
S_{n}^{\left(\kappa_{1}, \infty, \kappa_{3}, \kappa_{4}\right)}(x)=P_{n}^{(\alpha, \beta)}(x)+b_{n-1} P_{n-1}^{(\alpha, \beta)}(x), \quad n \geq 0,
$$

with $b_{-1}=b_{0}=0$.

It is well known that the sequence of monic Jacobi polynomials, $\left\{P_{n}^{(\alpha, \beta)}\right\}_{n=0}^{\infty}$, satisfies

$$
P_{n+1}^{(\alpha, \beta)}(x)=\left(x-\lambda_{n+1}^{(\alpha, \beta)}\right) P_{n}^{(\alpha, \beta)}(x)-\gamma_{n+1}^{(\alpha, \beta)} P_{n-1}^{(\alpha, \beta)}(x), \quad n \geq 1,
$$

with $P_{0}^{(\alpha, \beta)}(x)=1, P_{1}^{(\alpha, \beta)}(x)=x-\lambda_{1}^{(\alpha, \beta)}$,

$$
\begin{gathered}
\lambda_{n+1}^{(\alpha, \beta)}=\frac{\beta^{2}-\alpha^{2}}{(2 n+\alpha+\beta)(2 n+\alpha+\beta+2)}, \quad n \geq 0, \\
\gamma_{n+1}^{(\alpha, \beta)}=\frac{4 n(n+\alpha)(n+\beta)(n+\alpha+\beta)}{(2 n+\alpha+\beta-1)(2 n+\alpha+\beta)^{2}(2 n+\alpha+\beta+1)}, \quad n \geq 1 .
\end{gathered}
$$

Now, we can prove the following result.

Theorem 3.5. If the conditions of Lemma 3.1 are satisfied, $n \geq 3$ and $\kappa_{2}$ large enough, then the $n$ zeros of $S_{n}^{\left(\kappa_{1}, \kappa_{2}, \kappa_{3}, \kappa_{4}\right)}$ lie inside the interval $(-1,1)$ provided that

i) for $\kappa_{3} \leq-1, \alpha$ and $\beta$ are such that

$$
b_{n-1}<\frac{(2 n+\alpha+\beta+1)(2 n+\alpha+\beta)}{2 n(n+\alpha)} \gamma_{n+1}^{(\alpha, \beta)},
$$

ii) for $\kappa_{3} \geq 1, \alpha$ and $\beta$ are such that

$$
b_{n-1}>-\frac{(2 n+\alpha+\beta+1)(2 n+\alpha+\beta)}{2 n(n+\beta)} \gamma_{n+1}^{(\alpha, \beta)} .
$$


Proof. i) For $x=-1$ it is known that

$$
P_{n}^{(\alpha, \beta)}(-1)=-\frac{(2 n+\alpha+\beta+1)(2 n+\alpha+\beta)}{2 n(n+\alpha)} \gamma_{n+1}^{(\alpha, \beta)} P_{n-1}^{(\alpha, \beta)}(-1)
$$

and $\operatorname{sgn}\left(P_{n}^{(\alpha, \beta)}(-1)\right)=(-1)^{n}$. Then, from $(26)$,

$$
\begin{gathered}
S_{n}^{\left(\kappa_{1}, \infty, \kappa_{3}, \kappa_{4}\right)}(-1)=P_{n-1}^{(\alpha, \beta)}(-1) \\
\times\left(b_{n-1}-\frac{(2 n+\alpha+\beta+1)(2 n+\alpha+\beta)}{2 n(n+\alpha)} \gamma_{n+1}^{(\alpha, \beta)}\right) .
\end{gathered}
$$

Choosing $\alpha$ and $\beta$ such that

$$
b_{n-1}<\frac{(2 n+\alpha+\beta+1)(2 n+\alpha+\beta)}{2 n(n+\alpha)} \gamma_{n+1}^{(\alpha, \beta)},
$$

we get $\operatorname{sgn}\left(S_{n}^{\left(\kappa_{1}, \infty, \kappa_{3}, \kappa_{4}\right)}(-1)\right)=(-1)^{n}$.

From Theorem 3.4, for $\kappa_{3} \leq-1$, at most $s_{n, 1}$ lies outside $(-1,1)$. Since $S_{n}^{\left(\kappa_{1}, \infty, \kappa_{3}, \kappa_{4}\right)}$ is monic and $\operatorname{sgn}\left(S_{n}^{\left(\kappa_{1}, \infty, \kappa_{3}, \kappa_{4}\right)}(-1)\right)=(-1)^{n}$ then $s_{n, 1}>-1$ and all zeros of $S_{n}^{J_{2}}$ lie inside $(-1,1)$.

ii) For $\kappa_{3} \geq 1$, the proof is analogous using $x=1$.

Acknowledgements. This research was supported by grants from CAPES, CNPq and FAPESP of Brazil and by grants from Ministerio de Ciencia e Innovación (Micinn) of Spain and European Regional Development Fund (MTM2008-06689-C02-02) and by Junta de Andalucía (G.I. FQM 0229). The authors would like to thank the referees for their valuable remarks, suggestions and references.

\section{REFERENCES}

[1] E.X.L. Andrade, C.F. Bracciali and A. Sri Ranga, Zeros of Gegenbauer-Sobolev orthogonal polynomials: beyond coherent pairs. Acta Appl. Math., 105 (2009), 65-82.

[2] A.C. Berti and A. Sri Ranga, Companion orthogonal polynomials: some applications. Appl. Numer. Math., 39 (2001), 127-149.

[3] A.C. Berti, C.F. Bracciali and A. Sri Ranga, Orthogonal polynomials associated with related measures and Sobolev orthogonal polynomials. Numer. Algorithms, 34 (2003), 203-216.

[4] C.F. Bracciali, D.K. Dimitrov and A. Sri Ranga, Chain sequences and symmetric generalized orthogonal polynomials. J. Comput. Appl. Math., 143 (2002), 95-106. 
[5] T.S. Chihara, An Introduction to Orthogonal Polynomials. Mathematics and its Applications Series, Gordon and Breach, New York, (1978).

[6] M.G. de Bruin, W.G.M. Groenevelt and H.G. Meijer, Zeros of Sobolev orthogonal polynomials of Hermite type. Appl. Math. Comput., 132 (2002), 135-166.

[7] A.M. Delgado and F. Marcellán, Companion linear functionals and Sobolev inner products: a case study. Methods Appl. Anal., 11 (2004), 237-266.

[8] W.G.M. Groenevelt, Zeros of Sobolev orthogonal polynomials of Gegenbauer type. J. Approx. Theory, 114 (2002), 115-140.

[9] A. Iserles, P.E. Koch, S.P. Nørsett and J.M. Sanz-Serna, On polynomialsorthogonal with respect to certain Sobolev inner products. J. Approx. Theory, 65 (1991), 151-175.

[10] D.H. Kim, K.H. Kwon, F. Marcellán and G.J. Yoon, Zeros of Jacobi-Sobolev orthogonal polynomials. Int. Math. J., 4(5) (2003), 413-422.

[11] F. Marcellán, T.E. Pérez and M.A. Piñar, Gegenbauer-Sobolev orthogonal polynomials, in Nonlinear Numerical Methods and Rational Approximation, II, Math. Appl., 296 (1994), $71-82$.

[12] P. Maroni, Sur la suite de polynômes orthogonaux associée à la forme $u=\delta_{c}+\lambda(x-c)^{-1}$. Period. Math. Hungar., 21 (1990), 223-248.

[13] H.G. Meijer, Determination of all coherent pairs. J. Approx. Theory, 89 (1997), 321-343.

[14] H.G. Meijer and M.G. de Bruin, Zeros of Sobolev orthogonal polynomials following from coherent pairs. J. Comput. Appl. Math., 139 (2002), 253-274.

[15] G. Szegö, Orthogonal Polynomials, vol. 23 of Amer. Math. Soc. Colloq. Publ., $4^{\text {th }}$ ed., Amer. Math. Soc., Providence, RI (1975). 